\title{
Bericht zum „International Coaching in Leadership Forum ICLF“ (11./12. Oktober 2017) und der ,Annual Conference - Coaching in Leadership and Healthcare“" (13./14. Oktober 2017) des Institute of Coaching, McLean Hospital, Harvard Medical School Affiliate (Boston, MA, USA)
}

\author{
Robert Wegener $^{1}$
}

Online publiziert: 14. November 2017

(C) Der/die Autor(en) 2017. Dieser Artikel ist eine Open-Access-Publikation.

Die Verbreitung und Differenzierung von Coaching als Beratungsform des 21. Jahrhunderts schreitet rasch voran. Auch wenn sehr populär, so ist Führungskräfte-Coaching längst nicht mehr die einzig anerkannte Coaching-Form. Coaching von erwerbslosen Menschen oder Coaching im Gesundheitssektor - um nur zwei prominente Beispiele zu nennen - werden immer bedeutsamer und verweisen auf eine zunehmende Marktsegmentierung. Dabei folgt Coaching der Idee, Menschen - seien es Führungskräfte, Ärztinnen, Patienten oder erwerbslose Personen - in ihren Anliegen zu unterstützen. Das bedeutet, ihnen zu helfen, besser und erfolgreicher zu werden in dem was sie tun oder was sie tun möchten.

Die Etablierung von Coaching fusst auf einer wachsenden Gemeinschaft professioneller Coaches. Dabei handelt es sich um eine Community, die in ihrer Entwicklung und Identitätsbildung noch am Anfang steht. Genauso wie die Klienten und Klientinnen von Coaches durchläuft auch diese Gemeinschaft einen Entwicklungsprozess, um in der eigenen Identität zu reifen und ihrem professionellen Handeln erfolgreicher zu werden. Dieser Entwicklungsprozess bedarf u. a. kritischer Reflexion und eines konstanten Austausches. Wichtige Gefässe dieses Austausches sind zum einen Coaching-Verbände, die sich mit relevanten Qualitätsfragen beschäftigen. Weitere Möglichkeiten dieses Austausches sind Coaching-Weiterbildungen und damit verbundene Alumni-Treffen. Ein dritter Ort des Austauschs und

Robert Wegener

robert.wegener@fhnw.ch

1 Fachhochschule Nordwestschweiz, Institut Beratung, Coaching und Sozialmanagement ICSO, Hochschule für Soziale Arbeit, Riggenbachstraße 16, 4600 Olten, Schweiz der Entwicklung dieser Gemeinschaft sind Coaching-Tagungen.

Coaching-Tagungen mit internationalem Charakter und Kontinuität, die den Anspruch erheben dürfen, neben primär praxisnahen Entwicklungen auch grundlegende wissenschaftliche Erkenntnisse aufzugreifen und in den Coaching-Diskurs einzuspeisen, gibt es bereits einige, wozu die Folgenden zu den wohl wichtigsten zählen:

- „Annual Conference - Coaching in Leadership and Healthcare" des Institute of Coaching (USA) ${ }^{1}$

- „Coaching and Mentoring Research Conference“ der Oxford Brookes University (England) ${ }^{2}$

- „International Congress of Coaching Psychology“ der International Society for Coaching Psychology ${ }^{3}$

- „Internationaler Coachingkongress ,Coaching meets Research “" der Fachhochschule Nordwestschweiz (Schweiz)

- „Coaching Kongress“ der Hochschule für angewandtes Management FH (Deutschland) ${ }^{5}$

Im vorliegenden Bericht wird nun Einblick gegeben in eine der weltweit renommiertesten Coaching-Tagungen, die jedoch aus eigener Erfahrung vielen deutschsprachigen Coaches nicht bekannt ist. Dabei handelt es sich um die vom Institute of Coaching organisierten und jährlich in Boston (USA) stattfindenden „Coaching in Leadership and Healthcare" Konferenz sowie dem davor stattfindenden „International Coaching in Leadership Forum ICLF“. Bei-

\footnotetext{
${ }^{1} \mathrm{http} / / / \mathrm{www}$.instituteofcoaching.org/conferences-events/ioc-conference.

2 http://business.brookes.ac.uk/research/iccmsresearch-events/coachingmentoring-conference.

3 http://www.coachingpsychologycongress.org.

${ }^{4}$ http://www.coaching-meets-research.ch.

5 https://www.coaching-kongress.com/programm.
} 
de Veranstaltungen besuchte der Autor dieses Berichts im Oktober 2017.

\section{Institute of Coaching ${ }^{6}$}

Das Institute of Coaching (IOC) als Veranstalterin der genannten Anlässe wurde 2009 offiziell gegründet und gehört zum McLean Hospital der Harvard Medical School. Zum Gründerinnen- und heutigen Führungsteam gehören Dr. Carol Kauffman (director), Margaret Moore (co-director) und Dr. Susan David (co-director). Dotiert ist das Institut mit 2 Mio. US\$, gestiftet von der Harnisch Stiftung. Das Geld wird zur Förderung von Coaching-Forschung eingesetzt. Das Ziel des IOC ist es, Coaches zu unterstützen, Coaching als ,powerful vehicle for change“ weltweit zum Einsatz zu bringen. Dazu beschreibt das IOC folgendes Angebot zur Professionalisierung und Entwicklung der Coaching Gemeinschaft:

„We provide world-class professional development and community networking through our webinars, learning events, sell-out annual Coaching in Leadership and Healthcare Conference sponsored by Harvard Medical School, and an unparalleled coaching resource library. We also provide grants to support promising coaching research. ${ }^{\text {"7 }}$

Besonders am IOC, gerade auch im Unterschied zu den anderen Tagungs-Veranstaltern, ist das breite Angebot an Webinaren und Fachliteratur für Institutsmitglieder sowie die finanziellen Ressourcen für Coaching-Forschungsprojekte.

\section{International Coaching in Leadership Forum ICLF}

Das „International Coaching in Leadership Forum ICLF“, das jeweils vor der ,Coaching in Leadershop and Healthcare" Konferenz stattfindet, wurde am 11./12. Oktober 2017 zum 6ten Mal durchgeführt. Bei den etwa 40 Teilnehmern und Teilnehmerinnen handelt es sich um ,leading executive coaches from around the world who have deep experience in coaching c-suite executives from a broad array of industries“. Die Teilnahme an diesem exklusiven Anlass ist den Fellows sowie den Konferenzpartner des IOC vorenthalten. Ziel des Forums ist es, im direkten Austausch mit TopFührungskräften, aktuelle Herausforderungen und Anliegen dieser zu erkunden und zu klären, wie Coaches diesen die beste Unterstützung bieten können.

\footnotetext{
${ }^{6}$ Für einen vertiefen Einblick in das Institute of Coaching uns eine Angebote vergleiche http://www.instituteofcoaching.org/.

7 http://www.instituteofcoaching.org/about/mission-history.
}

Das Konzept des Forums ist einfach und überzeugend. So präsentieren und diskutieren jeweils eine Auswahl hochkarätiger Gäste ihre Positionen untereinander, bevor daran anschliessend die anwesenden Coaches in die Diskussionen eingebunden werden. Am Nachmittag werden Kleingruppen gebildet, in denen mit den Gästen vertiefende Gespräche geführt werden können.

Am ersten Tag des diesjährigen Forums waren John Brownstein (Chief Innovation Officer des Children's Hospital Boston), Joe Gammal (Managing Partner Synecticsworld, einem Beratungsunternehmen) und Susan Musinsky (Executive Director des Social Innovation Forums) auf dem Panel. Moderiert durch David Peterson (,Director Executive Coaching \& Leadership“ bei Google) diskutierten die Anwesenden, wie sie mit dem Anspruch permanenter Innovation und Wandel umgehen. Eine zentrale Erkenntnis war, dass es von Bedeutung ist, aus jeweils vorhandenen Ideen und Innovationen nur die wirklich besten auszuwählen. Darüber hinaus, und dazu herrschte breiter Konsens, bestand eine weitere Erkenntnis darin, dass Innovationen selbst nicht die besondere Herausforderung darstellen, sondern vielmehr deren Umsetzung, die entsprechende Ressourcen und viel Geduld sowie Ausdauer bedarf. Zudem besteht eine gegenwärtige Herausforderung darin, dass Organisationen und Führungskräfte nachhaltig innovativ werden, also nicht nur punktuell bei anstehenden Herausforderungen unterstützt werden. Dazu ist es wichtig zwischen operativen Geschäften und Innovationsprozessen klar zu unterscheiden und für die Innovationsprozesse entsprechende Ressourcen bereitzustellen.

Am zweiten Tag erhielten die anwesenden Coaches Einblick in die Perspektiven erfolgreicher CEOs. Teil des Panels waren Feyzi Fatehi (CEO von Corent Technology, einem Software Unternehmen), Pooneh Mohajer (CEO von tokidoki) und Joe Ostolaza (ein peruanischer Grossunternehmer) sowie Marshall Goldsmith (Top-Executive Coach und Beststeller-Autor). Neben den Einblicken in die Biographien, Tätigkeitsfelder und Herausforderungen dieser Persönlichkeiten waren es v. a. deren Antworten auf ausgewählte Fragen, die die Aufmerksamkeit der Anwesenden auf sich zog. So lautete eine der Fragen, welchen Rat sich die CEOs heute an eine jüngere Ausgabe von sich selbst geben würden. Die Antworten reichten von „Not to be scared and worry so much“ (P. Mohajer), „Have more courage and take more risk!“ (F. Fatehi), ,More openness for help“ (J. Ostolaza) bis hin zu „Do the hard things first!“ (D. Peterson). Auf die Frage, worauf die eigenen Erfolge gründen, nannten die meisten persönliche Vorbilder. Marshall Goldsmith, der mitunter mit Peter Drucker, dem Pionier der modernen Management-Lehre, arbeitete, beschrieb sein Erfolgsrezept so: „Hanging around with outstanding people makes you feel things are possible!“ 
Besonders und einzigartig am ICL-Forum war neben den persönlichen Gesprächen mit den hochkarätigen Gästen v. a. auch die Einzelgespräche mit den ebenso interessanten Coaches aus aller Welt sowie nicht zuletzt die vergnüglichen Mittagessen in der wunderschönen Pier-Gegend von Boston. Beendet wurde das Forum mit einem Empfang für die IOC Fellows und Konferenzpartner sowie zusätzlich bereits angereister Keynote Speakers (Anthony Grand (AUS), Reinhard Stelter (DK), Tatiana Bachkirova (UK)) der am Folgetag startenden Coaching-Konferenz.

\section{Annual Conference - Coaching in Leadership and Healthcare (13-14. Oktober 2017, Boston USA)}

Im vollständigen Kontrast zum ICLF als exklusivem Kleinevent ist die „Coaching in Leadership und Healthcare“ Konferenz ein beeindruckendes Grossevent mit rund 600 Teilnehmenden aus aller Welt, das im noblen, und damit zur Konferenz passenden, Renaissance Boston Waterfront Hotel stattfand. Gewidmet war die Konferenz dem jüngst verstorbenen Sir John Whitmore, einem der Coaching-Väter und Autor des Bestsellers Coaching for Performance, der zugleich einer der ersten Begleiter des IOC in seiner Gründungsphase war.

Die Eröffnungskeynote hielt der international renommierte Wissenschaftsjournalist Daniel Golemann, der mit seinem Buch „Emotionale Intelligenz“ weltweit bekannt wurde. Sein Vortrag beschäftigte sich mit der Frage der Integration von Achtsamkeit (,mindfulness") in Coaching sowie den positiven Effekten entsprechender AchtsamkeitsTechniken wie erhöhte Präsenz, Resilienz und Fürsorge.

Die zweite Keynote-Sprecherin war Bestseller-Autorin Susan Cain. Sie referierte über introvertierte Menschen „,in a world that can't stop talking“. Besonders an ihrem Vortrag $^{8}$ war ihr Plädoyer für mehr introvertierte Menschen in der Führung, da diese durch ihre besondere Willenskraft, ausgewiesene Hingabe und ruhige Art Unternehmen nachweislich erfolgreich(er) führen.

An diese Plenums-Keynotes anschliessend gab es über den restlichen Tag verteilt - wie auch am Folgetag - parallel stattfinden Keynotes (Dr. Anthony Grant, Dr. Ryan Niemic) sowie unterschiedliche Tracks $(1,5 \mathrm{~h}) \mathrm{zu}$ folgenden Themenbereichen:

- Leadership (Mindful Habit Change mit Daniel Goleman),

- Health \& Wellness (Implementing a Leadership and Executive Coaching Program mit Anthony Grant),

\footnotetext{
8 „Quiet: How to harness the strength if introverts to transform how we work, lead and innovate“.
}

- Positive Psychology (Boasting Flourishing, Fostering Resilience mit Ryan Niemic)

- Special Topics (Triggers mit Marshall Goldsmith)

- Research Symposium

In den Research Symposien „Health and Wellness" ${ }^{\star 99}$ und „Leadership Coaching" ${ }^{10}$ gab es jeweils vier Beiträge zu aktuellen Coaching-Forschungsprojekten.

Marshall Goldsmith überzeugte in seinem ThemenTrack durch seine äusserst unterhaltsame und lebendige Art, wobei er neben fachlich interessanten Impulsen zu seinem Coaching Approach - dem „Stakeholder Centred Coaching SCC“" zur Entwicklung von Führungskräften die Teilnehmerinnen und Teilnehmer einlud, eines seiner Coaching-Kernstücke, das „Feedforward“, im Austausch mit den Anwesenden direkt auszuprobieren. Darüber hinaus rief er dazu auf, jeden Tag einige Minuten dafür zu verwenden, um sich der Überprüfung selbst gesetzter Ziele zu widmen (,Daily Question Process“). Ein Prozess, den er selbst seit Jahren verfolgt und als höchst wirksam und zugleich im Sinne von Selbstkonfrontation als sehr hart bewertet. Gewissermassen als Krönung drückte er zum Workshopende den knapp 100 Anwesenden als Überraschung eine Hardcopy seines Best-Sellers „Triggers“ in die Hand.

Zum Abschluss des ersten Tags gaben Tony Mark (CEO der Library of New York) und Soren Schroder (CEO von Bunge) im versammelten Plenum offenen Einblick in ihre Erfahrungen als Coachees von Marshall Goldsmith. In beeindruckender Weise berichteten die beiden in aller Selbstverständlichkeit über ihre Schwächen als Führungskräfte sehr grosser Organisationen und damit verbundener Lernund Entwicklungsprozesse als Teil ihrer Coachings.

Der zweite Konferenztag begann mit einer Reihe an Preisverleihungen, u.a. an James \& Janice Prochaska für ihre Entwicklung und Verbreitung des „Transtheoretischen Modells“, das heute oft als veränderungstheoretische Grundlage im Coaching Einsatz findet. Weiter wurden

\footnotetext{
${ }^{9}$ Health and Wellness Coaching Research Symposium: (1) Gary Sforzo, PhD, „Health an Wellness Coaching Compendium: New Resource for Practitioners and Researchers“, (2) Ruth Wolever, PhD, „Generating Change: Health Coaching and Genetic Risk“, (3) Joel Edman, $\mathrm{PhD}$, „Effects of a Health Coaching Program in Chronic Disease“, (4) Alyssa McGonagle, Coaching workers with Chronic Health Conditions.

${ }^{10}$ Leadership Coaching Research Symposium: (1) Tatiana Bachkirova, $\mathrm{PhD}$, ,The Good Old Wine of Pragmatism in the New Bottles of Coaching and Coaching Research“, (2) Carrie Arnold, PhD, „The Silenced Female Leader: Coaching Women to find Purposeful Voice“, (3) Gail Greenstein, PhD, ,Got Privilege? What does it have to do with Executive Coaching? Let's talk about intersectionality: Race, class, gender variations, sexual orientation and multiple identities“, (4) Venkata Nanduri, $\mathrm{PhD}$, How the Behavioural Changes are sustained by the Manager-Coachees over time after Coaching ended.
} 
folgende Forscher und Forscherinnen für ihre Arbeiten ausgezeichnet:

- Best Poster Award - The Relationship between Working Alliance and Client Outcomes in Coaching: A Meta-Analytic Review, Carolin Grassman, International Management University, Berlin

- Honorable Mention Award - Development Coaching as co-regulating agent of professional identity: a new conceptual coaching framework integrating identity theory principles, Fance Asselin, University of Quebec, Canada

- Honorable Mention Award - Emerging Evidence for the effectiveness of coaching for attention deficit/hyperactivity disorder: a review of the literature, Elizabeth Ahmann, Maryland University of Integrative Health

Nach der Preisverleihung erfolgte eine Keynote von Dr. Margaret Wheatley. Der Titel ihres Beitrags lautete „Restoring Leadership as a Noble Profession“. In gewisser Weise Gegenstück zu der dominanten Vorstellung, Coaches müssten ihren Klienten und Klientinnen helfen, sich immer schneller zu verändern und noch agiler zu werden, rief Margaret Wheatley dazu auf, im Kontext einer krisenbelasteten Welt Führungskräfte darin zu unterstützen, sich als Fundament ihres Tuns an Werten wie Gewissenhaftigkeit, Menschlichkeit und Vernunft zu orientieren.

Im weiteren Verlauf des zweiten Konferenztags gab es erneut eine Vielzahl parallel laufender Keynotes (James Prochaska, „Coaching to Thrive“, Robert Waldinger, „What Science can tell us about the good life" und David Peterson „Executive Coaching on the Cusp of Disruption“) und Tracks zu unterschiedlichen Themen sowie Forschungsprojekten (u. a. eine Fortführung des Health and Wellness Coaching Research Symposium ${ }^{11}$ sowie des Leadership Coaching Research Symposium ${ }^{12}$ ).

Im Track von Deborah Helsing, EdD, einer der Nachfolgerinnen des renommierten Harvard Professors Robert Kegan, konnten die Workshop-Teilnehmenden einen interessanten und effektiven Veränderungsansatz - den „Immu-

${ }^{11}$ Health and Wellness Coaching Research Symposium: (1) Reinhard Stelter, $\mathrm{PhD}$, „The Health Coachg as Collaborative Partner“, (2) Kiyoshi Ando, MD, „Clinical Evidence Development and Education of Medical Coaching for Narrative Based Medicine in Japan“, (3) Leslie Schwab, MA \& Alyssa McGonagle, PhD, „Coaching for primary care physicians“, (4) Erin Pearson, PhD, ,Be Kind to yourself-because you're doing fine": Motivational Interviewing via CoActive Life Coaching as an intervention for improving physical and psychological health indices in first-time mothers.

12 Leadership Coaching Research Symposium: (1) Gordon Spence, $\mathrm{PhD}$, Sleeper Effects in Executive Coaching, (2) Anne Marie Halton, $\mathrm{PhD}$, Intentional Change Theory, Coaching and Leader Effectiveness, (3) Nicky Terblance, PhD, Using the transformative Transition Coaching Framework to Support Leaders during Career Transitions, (4) Janice Sabatine, $\mathrm{PhD}$, Strategies and two-year Results for a Vision-based Coaching Supplement to a Graduate Student Career Course. nity To Change“ Approach - direkt an einer eigenen Herausforderung und im Austausch mit den anderen Anwesenden ausprobieren. Im Kern dieses theoretisch und empirisch fundierten Modells steht die Vorstellung, dass Menschen sich mit ,guten Gründen“ in der Erreichung ihnen wichtiger Ziele im Wege stehen. Die Übertragung des Immunsystems auf die Psyche verweist auf die Vorstellung tiefliegender und bremsender Überzeugungen, die es zu identifizieren und schrittweise zu dekonstruieren gilt.

Abgeschlossen wurde der zweite Tag und damit die Konferenz insgesamt mit einem stimmungsvollen Konzert des Sängers und Gitarristen Livingston Taylor. Diesem gelang es, die Anwesenden in seinen musikalischen Bann zu ziehen und damit der Konferenz einen runden und originellen Abschluss zu geben.

\section{Eine persönliche Fazit}

Die beiden Anlässe des IOC, die ich als Autor dieses Beitrags besuchen konnte, waren von grossem Wert. Neben vielen interessanten fachlichen Impulsen war es vor allem auch die Möglichkeit, in den Austausch mit Gleichgesinnten aus der ganzen Welt zu gehen, was diesem internationalen Anlass eine besondere Note verlieh. So hatte ich die Chance, neben vielen Grössen der internationalen Coaching-Szene auch neue Kontakte zu knüpfen zu Kollegen und Kolleginnen aus den unterschiedlichsten Ländern. Damit verbunden gewann ich interessante Einblick in die Entwicklungen von Coaching etwa in China oder Brasilien. Leider wurde keine der Keynotes im Plenum von wirklich hartgesottenen Coaching-Forscher/innen gehalten wurde. Schade, da die Veranstalterin sich ja gerade Coaching-Forschung auf die Fahne geschrieben hat. Alles in allem kann ich aber neben kleineren Mängeln die Teilnahme an beiden Veranstaltungen des IOC als wichtiger Ort der Professionalisierung von Coaching und des Austauschs der Coaching-Community empfehlen. Damit verbunden auch den Blick auf die IOC-Website und den dort vorhandenen Angeboten (www.instituteofcoaching.org/). Die nächste "Coaching in Leadership and Healthcare Conference“ findet am 28-29 September 2018, erneut in Boston, statt.

Open Access Dieser Artikel wird unter der Creative Commons Namensnennung 4.0 International Lizenz (http://creativecommons.org/ licenses/by/4.0/deed.de) veröffentlicht, welche die Nutzung, Vervielfältigung, Bearbeitung, Verbreitung und Wiedergabe in jeglichem Medium und Format erlaubt, sofern Sie den/die ursprünglichen Autor(en) und die Quelle ordnungsgemäß nennen, einen Link zur Creative Commons Lizenz beifügen und angeben, ob Änderungen vorgenommen wurden. 